\title{
Estrategias y Mecanismos para la Construcción de una Cultura de Paz en la Educación Secundaria en Bogotá, Colombia
}

\section{Strategies and Mechanisms for the Construction of a Peace Culture in Secondary Education in Bogotá, Colombia}

Catalina Acosta Oidor *, Luz A. Tabares Rojas, Paula N. Castillo Acosta, M. Catalina López Andrade, Luisa F. Luque Ramírez, Ana M. Ortiz Arévalo y Nataly Vargas Rodríguez

Universidad Santo Tomás, Bogotá

\section{DESCRIPTORES:}

Educación para la paz

Cultura de paz

Jóvenes

Instituciones educativas

Colombia

\begin{abstract}
RESUMEN:
El presente artículo es resultado de una investigación sobre los mecanismos y estrategias promovidos en la educación secundaria para generar una cultura de paz en Colombia, una sociedad que busca transformar un contexto generalizado de conflicto armado en un ambiente donde primen las soluciones negociadas. Esta intención se manifiesta a través de la firma del acuerdo de paz en 2016, entre el gobierno de 2010 -2018 y la guerrilla FARC. La sociedad experimentó desde la segunda mitad del siglo XX las consecuencias de este conflicto, evidenciadas en la priorización de la violencia como mecanismo para gestionar las diferencias. Por esta razón, se hace necesario evidenciar las formas en las que se prepara a la sociedad para un ambiente pacífico. Uno de los escenarios principales para ello es sin duda el sistema educativo -escuelas, colegios, universidades-. En el presente caso se estudia la experiencia de dos colegios: IED Antonio José Uribe y Santa Francisca Romana de Bogotá. A través de una metodología mixta que involucró encuestas, entrevistas y talleres, se evidenció cómo la paz y la violencia son dos ejes presentes en la vida cotidiana y no necesariamente se restringen a consideraciones sobre lo que ocurre a nivel político.
\end{abstract}

\section{KEYWORDS:}

Peace education

Peace culture

Young people

Schools

Colombia

\section{ABSTRACT:}

The following article is the result of an investigation about mechanisms and strategies applied in high school education to generate what 's known as "peace culture in Colombia", a society that seeks to transform a generalized context of armed conflict into one were negotiated solutions are the first choice. The intention of transforming this context was manifested in the peace treaty of 2016 between the Colombian Government (2010-2018) and FARC guerrilla. During the second half of the twentieth century, society has experienced the consequences of this conflict, which are evidenced in the prioritization of violence as a mechanism to mediate differences. Because of this, it is necessary to display the different ways in which society is being prepared to live in a peaceful environment. One of the most important areas to accomplish this is the education system (pre-school, middle school, high school, College, university etc.). In the following research two schools and their experiences are studied (IED Antonio José Uribe and Colegio Santa Francisca Romana located in Bogotá) using mixed methods such as, surveys, interviews and workshops. The results showed how peace and violence are two aspects present in the daily life and not necessarily narrowed down to politics.

CÓMO CITAR:

Acosta Oidor, C., Tabares Rojas, L., Castillo Acosta, P. N., López Andrade, M. C., Luque Ramírez, L. F., Ortiz Arévalo, A. M. y Vargas Rodríguez, N. (2021). Estrategias y mecanismos para la construcción de una cultura de paz en la educación secundaria en Bogotá, Colombia. Revista Internacional de Educación para la Justicia Social, 10(1),245-258. https://doi.org/10.15366/riejs2021.10.1.015

*Contacto: catalinaacosta@usantotomas.edu.co

ISSN: 2254-3139

revistas.uam.es/riejs
Recibido:

20 de octubre 2020

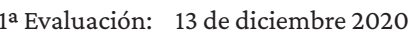

2a Evaluación: 9 de marzo 2021

Aceptado: $\quad 7$ de abril 2021 


\section{Introducción}

La historia colombiana de los siglos XX y XXI se ha caracterizado por la presencia constante del conflicto político armado entre el Estado y organizaciones al margen de la ley (guerrillas, narcotraficantes, paramilitares, Grupos Armados Organizados, etc.). Esta continuidad del conflicto armado, especialmente desde los años 60 , implica su priorización en el presupuesto nacional por encima de aspectos económicos, sociales y culturales, claves para el desarrollo del país.

Adicionalmente, los gobiernos han determinado el exterminio de estos actores, construyéndolos como un enemigo común a todos los colombianos, subvalorando la negociación y el consenso como forma de zanjar las diferencias. De esta manera, se promociona la violencia, e incluso la muerte, como la forma efectiva de resolver los desacuerdos y/o hacer justicia. Esta forma de pensamiento se puede extender de manera generalizada a relaciones cotidianas en la familia, la escuela y el barrio, perdiendo de vista la valoración del otro como semejante, con el que se debe convivir.

A pesar de que esta situación constituye de forma evidente un problema para la sociedad, sólo se percibe la necesidad de reflexionar y tomar medidas sobre ello cuando se experimenta la posibilidad de un escenario de posguerra. En este sentido, se dio entre 2012 y 2016, el Acuerdo de Paz con la guerrilla de las FARC.

Aunque la cifra de muertes en enfrentamientos en los que participaba las FARC disminuyó progresivamente entre 2012 (462) y 2017 (0) (Colombia 20/20, 2018), el proceso de paz no fue bien recibido por una gran parte de población que seguía viendo en las FARC un enemigo con el que no se debía negociar. Evidencia de esto, fue el resultado del plebiscito realizado en 2016, con el que se esperaba refrendar la primera firma del Acuerdo realizada el mismo año; el 50,21\% de la población sufragante rechazó la negociación frente a un $49,78 \%$ que le dijo "sí".

La evidencia de esta percepción cerrada a valorar los consensos como forma de alcanzar una convivencia pacífica, hace necesario preparar a la sociedad colombiana para la situación actual, en la que quienes deben suponerse ahora "antiguos" enemigos retornen a la vida civil y política de forma legal; esto implica dar a conocer las causas del conflicto armado, sin evidenciar posiciones a favor o en contra, e instaurar en la cotidianidad la importancia de la resolución pacífica de los conflictos.

Uno de los escenarios considerados para desarrollar esta tarea es el sistema educativo; por tal razón, el Gobierno Nacional empezó a promover la obligatoriedad de la Cátedra de Paz en las instituciones de educación primaria, básica y media, a través de la Ley 1732, decreto 1038 de 2015. Allí se indica que

La Cátedra de la Paz deberá fomentar el proceso de apropiación de conocimientos y competencias relacionados con el territorio, la cultura, el contexto económico y social y la memoria histórica, con el propósito de reconstruir el tejido social, promover la prosperidad general y garantizar la efectividad los principios, derechos y deberes consagrados en la Constitución. (Decreto 1038, 2015, Artículo 2)

Igualmente, se indica la cultura de paz como uno de los elementos principales en los que se debe enfocar el aprendizaje, el diálogo y la reflexión propiciados en este espacio, y esta se entiende como "el sentido y vivencia de los valores ciudadanos, los Derechos Humanos, el Derecho Internacional Humanitario, la participación democrática, la prevención de la violencia y la resolución pacífica de los conflictos". (Decreto 1038, 2015, Artículo 2)

Sin embargo, la falta de precisión de la normativa frente a contenidos, espacios de aplicación, capacitación docente y, en general, frente a las limitaciones de una cátedra para la generación de transformaciones más allá del contexto específico de la clase, hace necesario evaluar a través de qué tipo de estrategias y mecanismos los colegios ejercen una educación para la generación de una cultura de paz en un contexto de posguerra. La presente investigación versa precisamente sobre esta cuestión. 


\section{Estado del arte}

A partir de un ejercicio bibliométrico en Scopus se pudo observar que las investigaciones realizadas al respecto se ubican en dos grandes temas: educación para la paz y cultura de paz. En el primer caso, entre 2011 y 2018 se evidencia un aumento progresivo de investigaciones publicadas, pasando de un artículo por año hasta tres o cuatro en 2014, 2016 y 2018, y se abordan experiencias referentes a Brasil, Colombia y España. De otro lado, las investigaciones que abordan el tema de cultura de paz, se concentran en los años 2015 y 2016 con 5 y 4 artículos respectivamente, reduciéndose a dos artículos por año entre 2017 y 2018, y las experiencias abordadas se concentran en Brasil, Colombia, España y Norteamérica.

De estos estudios se destacan, en primer lugar, aquellos que centrados en el papel de los medios de comunicación como herramienta para la construcción de paz (Castellano et al., 2017; Moreira et al., 2016; Santos, 2016; Vargas, 2016) Desde esta mirada se busca mostrar cómo los medios masivos de comunicación funcionan como sustentadores del sistema hegemónico, y, por ende, afianzan las representaciones en las que se apoyan los conflictos. No obstante, dado su papel, también tienen la capacidad para generar espacios de convivencia pacífica y para la resolución de dichos conflictos. Castellano y otros (2017) analizan los medios alternos de empresas y universidades colombianas, resaltando la importancia de hacer uso de estos para afianzar valores y principios para gestionar las diferencias.

Por otra parte, Moreira y otros (2016), en el caso del acuerdo de paz entre Ecuador y Perú en 1998, evidencian la fuerza que los medios tienen en expandir interpretaciones de los conflictos de acuerdo con las relaciones de poder establecidas, por lo que se hace un llamado a la objetividad y a la concientización sobre la relación que deben instaurar con las ONG y la sociedad civil. Santos (2016), Vargas (2016) y García (2014) centran la atención en el papel de las TIC en la promoción de escenarios de paz en el aula, que de manera general se encuentran poco vinculadas en la intervención de los conflictos; allí se resalta la importancia de extender el papel de la universidad frente a la implementación de una cultura de paz desde las TIC para llegar a la sociedad en general. En el último caso, se resalta la importancia de la capacitación mediática e informática al profesorado; de las bibliotecas, archivos y otros proveedores de información; las condiciones de trasmisión de información; y la evaluación los contenidos y servicios que se promueven.

En segundo lugar, se encontraron estudios que evalúan la educación para la paz en la educación primaria, secundaria y superior. Carvalho y otros (2019), por ejemplo, analizan los castigos corporales y morales ejercidos por largo tiempo en la educación escolar brasilera, que reflejan la falta de respeto a la dignidad humana por cuenta de políticas educativas autoritarias. Por esta razón, los autores señalan la importancia de construir políticas educativas que se basen en los derechos humanos.

En otros casos, la educación para la paz está integrada al área de artes, como resalta Ramírez (2017) específicamente desde la educación musical. Sin embargo, la autora considera que estas áreas son relegadas en el currículo por cuenta de las políticas neoliberales que lo condicionan a la obtención de resultados empresariales; se fomenta el individualismo, el "solipsismo" y la competencia, olvidando a la persona y su dimensión social. En este sentido, la educación musical se considera un medio para conseguir la transformación estructural con procesos culturales que construyen espacios de paz.

De igual manera, Martínez (2017) analiza la educación para la paz en el pregrado de lengua inglesa en una institución española; donde las destrezas requeridas en dicha disciplina (habla, escucha, lectura, escritura e interacción permanente) son vistas como herramientas para promover competencias sociales como la cooperación, la comunicación o la resolución efectiva de conflictos.

Por otra parte, el análisis de la promoción de una cultura de paz se hace también a partir del estudio de los libros de texto implementados en la educación primaria, en el marco de la asignatura de “educación para la ciudadanía” (Sánchez y Vargas, 2017; Boqué et al., 2014). Sin embargo, se trata de un espacio académico que poco a poco va desapareciendo del currículo, lo que supone un freno a la formación en valores necesaria para generar relaciones pacíficas. Adicionalmente, se observa la ausencia del vocablo "paz" en la mayor parte de los textos, atención en el pasado y el futuro, prácticas participativas y democráticas y el olvido de los conflictos sociales y sus actores. 
En tercer lugar, se resaltan las investigaciones que buscan discutir la noción de cultura de paz como categoría de análisis. Moreira y Branco (2016), señalan la cooperación y resolución de conflictos como elementos centrales de la construcción de paz, a partir de un análisis de las percepciones de miembros de la policía militar brasilera. Se destaca de este estudio el reconocimiento de la distancia entre la imagen proyectada por los oficiales y su relación con la promoción de paz en los contextos donde trabajan. Salazar y Boschi da Silva (2014), también abordan la construcción discursiva de la noción cultura de paz, pero a partir del análisis de la cartilla "Cultura de paz, redes de convivencia", que tuvo amplia difusión en Brasil después de su publicación en 2009; resaltan cómo esta categoría conceptual resulta de un trabajo de producción de consenso que demandó esfuerzos colectivos para administrar las múltiples interpretaciones y la diversidad de posiciones al respecto.

En este mismo sentido, y para efectos de la presente investigación, se resalta la reseña de Cepeda (2016), sobre el Manual de construcción de paz publicado en México en 2015, que analiza la cultura de paz como un proceso que se desarrolla entre acciones y pensamientos. Así, la educación para la paz debe contemplar valores como la justicia, el respeto, la cooperación, la solidaridad y el compromiso con la alteridad, ello implica la identificación de las manifestaciones de los diferentes tipos de violencia (p.412).

Las tipologías de violencia son desarrolladas por el sociólogo Johan Galtung (2016), y corresponden a la violencia directa, expresada en actos de agresión; la violencia estructural, reflejada en las desigualdades de la estructura social y el sostenimiento de relaciones de dominación entre grupos; y la violencia cultural, referida a los elementos de la esfera simbólica que legitiman o justifican los otros tipos de violencia. De esto modo, se constituye un triángulo vicioso de violencias, pues allí se establecen relaciones complejas y multidireccionales, que permiten explicar la aparición y transformación de un tipo de violencia a partir de los otros y viceversa; pasando de relaciones causa-efecto a nivel histórico a retroalimentaciones en sucesos específicos; donde, por ejemplo, una disputa violenta entre grupos puede masificarse desarrollando formas de naturalización de esa relación e instaurando expresiones cotidianas de la misma, o una agresión puede ser la manifestación de la desigualdad en la estructura reflejada a partir de ideas discriminatorias.

Retomando a Cepeda (2016), en términos prácticos y en el nivel educativo, el Manual enfatiza en la necesidad de buscar metodologías, pedagogías y estrategias que tengan como eje el desarrollo de la autoconciencia, la responsabilidad y el reconocimiento del otro como semejante. Así, al referirse a construcción de paz, se parte según la autora, de una noción de paz positiva, desde el enfoque propuesto por Galtung, donde la paz negativa corresponde a la eliminación de la guerra y la violencia directa, mientras la paz positiva al combate de una violencia estructural y por ende la búsqueda de una justicia social.

En consonancia con todo lo anterior, y desde Colombia, Sánchez (2015) da cuenta de la relación entre cultura de paz y educación para la paz recogiendo los aportes teóricos de autores como Edgar Morin, Tuvilla Rayo, Jiménez Bautista, Francisco Muñoz, Vincent Fisas, Johan Galtung y Xesús Jares. Desde la conjunción de estos diferentes planteamientos es posible entender que la paz tiene diferentes dimensiones personales y sociales, y que es un camino (no un estado) cuya utilidad cosiste en gestionar los conflictos inherentes a los social sin recurrir a la violencia. En este sentido, la educación para la paz implica hacer uso de metodologías de enseñanza que fortalezcan la cultura de paz, entendida como el conjunto de elementos simbólicos que permite "regir las actuaciones sociales de los sujetos, orientándolos hacia la construcción de una sociedad más justa, solidaria y pluralista” (p. 75).

En esta misma línea, el presente artículo parte de reconocer que en Colombia la estructura social y ciertos rasgos culturales han posibilitado la generación de una normalización de formas de comportamiento violentas en la gestión de las diferencias, y es por ello que urge conocer cómo se aborda en el contexto escolar la transformación de dichas percepciones.

\section{Método}

Con el fin evaluar las estrategias y mecanismos de los colegios para la generación de una cultura de paz se llevó a cabo un estudio descriptivo. De este modo, se proporciona un diagnóstico de cómo las 
instituciones de educación secundaria materializan la Cátedra; el enfoque del estudio es mixto, pues se integran datos cuantitativos y cualitativos.

En primer lugar, se construyó una encuesta que tuvo como objetivo conocer a la población de estudio, sus principales problemáticas y sus percepciones frente al conflicto armado y a la concepción sobre la paz. Este cuestionario de 26 preguntas, se aplicó a 70 estudiantes de una institución pública (Institución Educativa Distrital Antonio José Uribe en adelante IED-AJU) ubicada en un barrio de estrato bajo, y a 58 estudiantes de una institución privada (Colegio Santa Francisca Romana) ubicada en un barrio de estrato alto. La población se seleccionó por vía de un muestreo por conveniencia, debido a la dificultad para el acceso a información referida a población en edad escolar. El procesamiento de la información obtenida se realizó a través del software estadístico Spss.

En segundo lugar, se realizaron talleres siguiendo la metodología de "juego de roles", que estuvieron enfocados en identificar el conocimiento y percepción de la población estudiantil en materia de temas relacionados con el conflicto político, el Acuerdo de Paz y la Cátedra de Paz, y en reflexionar junto a los estudiantes sobre su papel en la sociedad.

Adicionalmente, en la primera institución fue posible realizar cinco entrevistas a docentes (2), directivos (1) y estudiantes (2), que estuvieron enfocadas en indagar sobre la implementación de la Cátedra de Paz y en la percepción sobre la presencia de elementos de la cultura de paz y la educación para la paz en el contexto del colegio.

\section{Resultados}

\subsection{Contexto y caracterización de la población}

La IED-AJU se encuentra ubicada en el centro de la ciudad de Bogotá, en el límite de los barrios Las Cruces y San Bernardo, los cuales están clasificados por la Secretaría Distrital de Planeación (2019) como barrios con población en estratos 1 y 2 . Aunque ambos son considerados sectores tradicionales por ser parte del conjunto de los primeros barrios de la ciudad (Álvarez, 2016), actualmente se perciben como zonas que presentan deterioro físico y problemáticas sociales como microtráfico y zonas de consumo de drogas, pandillas, prostitución, y flujo constante de población habitante de calle. Estas problemáticas se relacionan con la ausencia de residentes permanentes en algunas calles por cuenta de la migración de los antiguos dueños de las viviendas, quienes las vendieron, y sus nuevos dueños reemplazaron su uso por talleres de mecánica, almacenes de muebles, locales comerciales, cantinas e inquilinatos o "paga diarios". (Navia, 1998; Serrano, 2015). Precisamente por la presencia de "paga diarios", ambos barrios son receptores de población desplazada de diferentes zonas del país, tanto por cuenta del conflicto armado como por razones económicas.

Adicionalmente, en mayo del año 2016, por iniciativa de la Alcaldía Mayor de Bogotá, se llevó a cabo el desalojo de un sector contiguo conocido como "El Bronx", donde residían personas drogodependientes y habitantes de calle, motivados por la presencia de expendios de sustancias psicoactivas (El Tiempo, 2016). Como resultado de dicho operativo, la población se dispersó a los barrios aledaños, entre ellos San Bernardo y Las Cruces.

De acuerdo con un docente de la IED-AJU, el contexto geográfico mencionado se refleja en la composición de su población estudiantil y las problemáticas que se presentan al interior de la misma, debido a que su población proviene de los barrios mencionados, pero también de otros aledaños como La Favorita y Santa Bárbara, los cuales presentan problemáticas semejantes. Es decir, que a la institución educativa acuden los hijos e hijas de las trabajadoras sexuales, de los expendedores de drogas; pero también de población desplazada en condiciones de pobreza, y como parte de la misma, miembros de comunidades indígenas.

Yo me atrevo a decir que hay tres ollas alrededor del colegio. No solamente están los niños y jóvenes de toda la población vulnerable, de trabajadoras sexuales, jíbaros, de "sayayines", de indigentes, de recicladores, etc. Sino que al colegio llegan desplazados de toda Colombia. Llegan de la Costa Atlántica, la Costa Pacífica, de los Llanos, Boyacá, Santanderes, el sur. Llegan a estos barrios porque 
acá hay "paga diarios", son sitios donde el arriendo es muy barato (...). Hay desplazados de toda Colombia, y el 30\% de nuestra población son indígenas: Emberá, pijaos, nasa, etc. (Docente de Artes, comunicación personal, 19 de octubre de 2018).

La población encuestada, estudiantes de los grados octavo (38) y noveno (33), reside en los barrios Las Cruces (46,4\%), San Bernardo (21,1\%) y Santa Bárbara (7\%) y, en consecuencia, los estratos más registrados son el $2(52,11 \%)$ y el 1 (7\%) (Figura 1$)$. Se resalta, además, un 32,3\% de estudiantes que no respondieron a esta pregunta, posiblemente por desconocimiento respecto al estrato del sector donde residen. Además, aunque un $69 \%$ de los estudiantes reconocen como ciudad de origen a Bogotá, un $29,5 \%$ de la población proviene de otras ciudades.

Por otro lado, el colegio Santa Francisca Romana “Las Pachas”, institución femenina con orientación católica y bilingüe, está ubicado en el barrio Las Margaritas, clasificado por la Secretaría Distrital de Planeación (2019) como barrio con población en estratos 5 y 6. La población encuestada corresponde a estudiantes de grado 11, quienes provienen de los barrios aledaños, ubicados en las localidades de Usaquén, Suba y Chapinero. Se trata en su mayoría (72\%) de zonas clasificadas con estratos 4,5 y 6 , caracterizadas por una óptima infraestructura vial, cercanía a centros comerciales, centros de servicios, zonas verdes y zonas predominantemente residenciales (El Tiempo, 1992; Portafolio, 2010).

En cuanto a su lugar de origen, se trata de una población proveniente principalmente de la ciudad de Bogotá (91,3\%). Los casos en los que se registra un lugar de origen diferente corresponden a otros países, tales como Estados Unidos y Ecuador. Aunque este hecho puede significar una experiencia de migración o desplazamiento, podría decirse que no se trata de razones de carácter estructural como las relativas al conflicto armado, sino más bien a episodios biográficos o mejoras en la calidad de vida.

Figura 1

Estrato de los estudiantes de IED-AJU y Colegio Santa Francisca Romana

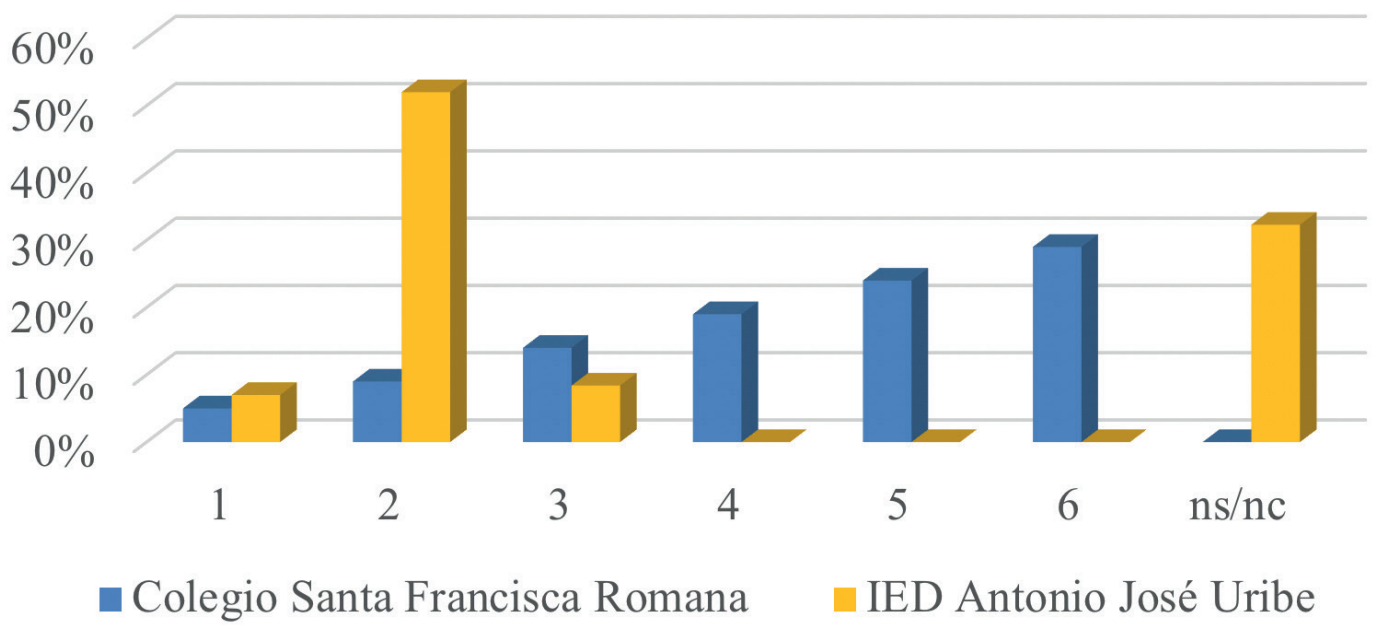

Por otra parte, en cuanto a la edad registrada por los estudiantes de ambos colegios, se evidencia un contraste que podría dar cuenta de una posición más favorable, en términos del origen social de las estudiantes de grado $11^{\circ}$ del colegio Santa Francisca Romana, dado que se encuentran entre los $16(3,4 \%), 17$ $(84,4 \%)$ y $18(12 \%)$ años; mientras en el caso del IED AJU, pese a que se trata de estudiantes de octavo y noveno grado el 63,2\% tienen edades entre los 15 y los 18 años. Teniendo en cuenta la proyección en años esperada por cada ciclo escolar según el Ministerio de Educación-Secundaria (11 a 14), Media (15 a 16) y Superior (17 en adelante)- se podría reconocer la existencia de un rezago en la edad de graduación para más de la mitad de la población encuestada en este último caso (Figura 2). 
Figura 2

Edad de los estudiantes del IED Antonio José Uribe $\left(8^{\circ}\right.$ y $\left.9^{\circ}\right)$ y del colegio Santa Francisca Romana $\left(10^{\circ}\right)$

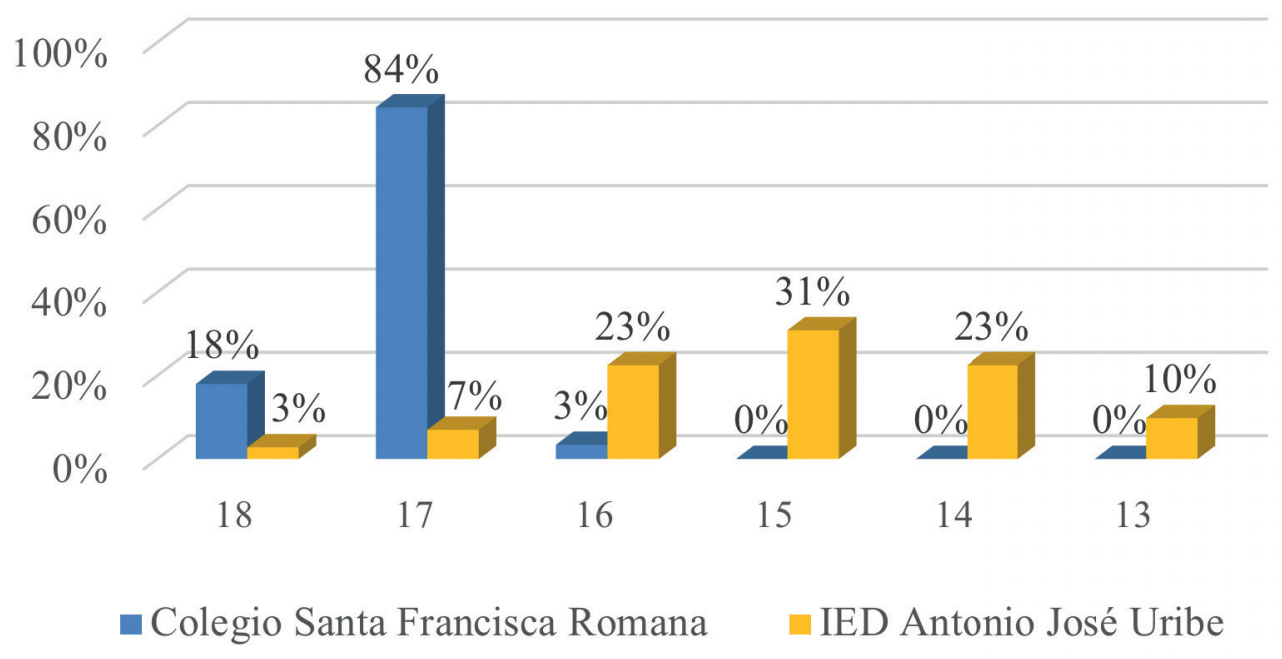

Adicionalmente, mientras en esta institución el 14,7\% de los encuestados manifiestan tener una doble condición, en tanto estudian y trabajan, la población del colegio Santa Francisca Romana en ningún caso registra una actividad laboral que se sume a su condición de estudiantes.

De otro lado, los estudiantes del IED-AJU viven en hogares donde la autoridad es ejercida principalmente por la madre (65\%) que es al mismo tiempo quién más asume los gastos (58\%), en contraste con los hogares en los que el padre asume la autoridad (17\%) y asume los gastos (28\%). De manera general, son madres y padres que tienen educación secundaria (39\%) y primaria (32\%) y técnica o tecnológica (7\%). En el caso de las estudiantes del colegio Santa Francisca, estas provienen de hogares donde la autoridad es ejercida por el padre (57\%) y/o padrastro (15\%), y en una proporción menor por la madre (26\%). Sin embargo, los gastos son asumidos por madre y padre en la mayoría de los casos (55\%), y en menor medida por el padre (20\%) y la madre (5\%) de forma individual. En cuanto a la escolaridad, se trata de madres y padres con estudios de posgrado (74\%) y pregrado (22\%) principalmente.

Finalmente, cuando se les interroga sobre su contexto barrial, quienes estudian en el IED-AJU manifiestan interés en cambiar de barrio $(75,7 \%)$ principalmente por cuenta de problemáticas como la inseguridad $(51,4 \%)$ y el consumo y venta de estupefacientes $(35,7 \%)$. En contraposición las estudiantes del colegio Santa Francisca Romana en su mayoría $(67,2 \%)$ manifiestan interés en continuar residiendo en los barrios actuales. Quienes manifiestan interés en cambiarse de barrio señalan como principales motivaciones los problemas de movilidad (17,2\%), espacios físicos deficientes (15,5\%), la inseguridad (15,5\%) y la mala convivencia $(8,6 \%)$ principalmente.

A partir de este contraste en las características sociodemográficas y del contexto familiar y barrial de la población encuestada, y teniendo en cuenta las teorías de la reproducción en el contexto escolar, se puede afirmar que la posición social de origen, los bienes materiales, cierto tipo de conocimientos y la legitimidad de los mismos, son factores de diferenciación que facilitan el éxito escolar, o que, por el contrario, lo obstaculizan cuando no se poseen. Se trata de elementos propios de sectores sociales con ventajas económicas, porque la cultura escolar y los contenidos de la enseñanza, responden a las formas del lenguaje y la cultura de éstos (Bourdieu y Passeron, 1979). De esta manera, las y los estudiantes del IED AJU que evidencian condiciones socioeconómicas desventajosas, que provienen de contextos vulnerables motivados por el conflicto armado o por situaciones de pobreza, y que en algunos casos asumen una doble condición como estudiantes y como trabajadores, tienen más dificultades para revertir las condiciones de sus hogares de origen. De igual forma, los estudiantes del Colegio Santa Francisca Romana, que presentan condiciones favorables, medidas por la estratificación de sus lugares de residencia, la edad escolar según el grado que cursan, la dedicación completa a la condición estudiantil y el grado de formación de sus padres; reproducen su posición en tanto se les facilitan el proceso de aprendizaje y el éxito al interior de la escuela y en la sociedad en general. 
Sin embargo, desde la postura teórica que aquí se adopta, es necesario considerar que desde las condiciones de desigualdad también se gestan las oportunidades para promover y generar el cambio social. Desde esta perspectiva, quienes provienen de entornos menos privilegiados no aceptan de forma pasiva la autoridad escolar y las desigualdades ocultas en el proceso de enseñanza, sino que, por el contrario, son sujetos activos que luchan contra dichas condiciones que se configuran como un destino social (Willis, citado por Giddens, 2004). Al mismo tiempo, quienes provienen de entornos ventajosos, tampoco pueden ser considerados como sujetos pasivos, pues su posición posibilita la comprensión de la dinámica de funcionamiento del orden y la concientización frente a la desigualdad que subyace al mismo. No obstante, dichas posiciones inciden en la forma en que se percibe el contexto sociopolítico y cómo se asumen temas aparentemente transcendentales para la sociedad en general, como la idea de una cultura de paz.

\subsection{La implementación de la Cátedra de Paz}

Las posibilidades de transformación de la sociedad colombiana, tanto a nivel estructural como en el plano de las relaciones cotidianas, pasan necesariamente por el proceso educativo. Para ello deben generarse contenidos y estrategias pedagógicas que involucren el reconocimiento de las condiciones generadoras de la desigualdad. Por esta razón, se gestionaron políticas educativas materializadas en normativas según las cuales se establece La Cátedra de la Paz como iniciativa para generar ambientes más pacíficos desde las aulas de Colombia, mas no se indican sus elementos más concretos y prácticos.

Pese a ello, la aproximación con la población educativa en los colegios objeto de la investigación, muestra importantes hallazgos con relación a las percepciones de los estudiantes sobre la realidad social y política, y especialmente frente a sus intereses en el marco de lo que les significa la idea de paz.

Así, por ejemplo, en el caso del IED-AJU, la Cátedra de Paz se encuentra vinculada a la asignatura de artes, ciencias naturales y ciencias sociales. En la primera por iniciativa del docente encargado y en las dos últimas por un acuerdo entre los docentes del colegio debido a que su implementación es evaluada por la Secretaría de Educación. Pese a ello, los docentes manifiestan que no se imparte una directriz sobre los contenidos que deben proporcionar en los espacios académicos a los que dicha cátedra se vincule. En cuanto a estos contenidos, los docentes resaltan la necesidad de enfatizar en temas centrales como la construcción de memoria histórica sobre el conflicto político armado y el Acuerdo de Paz firmado recientemente, con el propósito de evitar la naturalización de los acontecimientos violentos ligados a este conflicto.

La única área a la que está vinculada la Cátedra de Paz es a la de artes, sin embargo, hay unas clases de profundización que es un tema en el cual se decide profundizar, en el colegio, por un lapso de tiempo determinado, en donde trabaja un profesor de ciencias sociales y uno de ciencias naturales. Estas clases de profundización se les dictan solamente a algunos cursos, no todos los cursos la tienen, y la tienen cada semana con una intensidad horaria de dos horas. (...) el colegio no se ha organizado para darle realmente atención a la Cátedra de la Paz (...) cuando, en algún momento, la Secretaría de Educación ya empieza a pedir evidencia de que el colegio está implementando la Cátedra de la Paz, empiezan a buscar como locos qué profesores estaban trabajando el tema para entregar esas evidencias. (...) los profesores que están implementando el tema de la Cátedra de la Paz lo hacen porque decidieron por iniciativa trabajar los temas. (Docente orientadora de bachillerato, comunicación personal, 25 de noviembre de 2018)

Hay varios contenidos de los que se habla en la Cátedra para la Paz (...) uno que me parece vital para cualquier contexto, cualquier docente o población es lo de memoria histórica, porque si usted no tiene memoria histórica de lo que ha ocurrido acá con el conflicto armado interno y lo que sigue ocurriendo, pues usted no puede esperar que mágicamente todo se va a arreglar por firmar un papel. Entonces acá en Colombia pasa algo muy muy fuerte y es el olvido, acá en cuatro años a la gente se le olvida lo que se ha hecho y lo que no se ha hecho. (Docente de artes, comunicación personal, 19 de octubre de 2018)

Por otra parte, en el colegio Santa Francisca, los docentes han acogido la Cátedra de Paz como un contenido transversal a las diferentes asignaturas, como evidencia de su relevancia, de tal manera que se reconoce la presencia activa de contenidos ligados a la construcción de paz como una tarea asumida institucionalmente. Aunque no se plantea ninguna crítica a la falta de instrucción oficial por parte del Ministerio de Educación o 
de la Secretaría de Educación distrital, los docentes manifiestan que la forma de impartir los contenidos obedece a una concepción institucional sobre lo que consideran debe tratarse a la hora de hablar de paz.

Aquí en el colegio, el año pasado, se hizo un diagnóstico que sale de grupos focales con los estudiantes, con los docentes, con los padres de familia y el tema central es la cuestión de la violencia en la escuela y las relaciones entre los pares, que finalmente los dos están muy conectados. Los niños perciben que en el colegio hay mucha violencia, hay mucha agresión "por cualquier cosa". Hay violencias también, construidas culturalmente acerca de a quiénes les puedo agredir. Entonces, los niños agreden a las niñas, los grandes agreden a los pequeños, agresión entre familias dentro del colegio, entre hermanos, por ejemplo. Esas violencias están asociadas a las formas de interrelación a las que se han acostumbrado (...). (Docente orientadora de bachillerato, comunicación personal, 25 de noviembre de 2018)

(...) Las ollas de San Bernardo siempre entran en conflictos de territorio, entonces imagínese que ahíllegan chicos de los dos barrios y se encuentran ahi. Entonces muchas veces los problemas que ocurren entre estudiantes se vuelven problemas entre familias o entre pandillas (...) se necesita es que llegue Secretaria de Integración Social, Secretaría de Gobierno, que lleguen allá a ayudar. Como le digo, si allá el señor o el papá del chico no va a ir a ganarse medio mínimo cuando en la olla se gana un millón, es así de simple, y asimismo, aparte de esas rencillas por las bandas que hay ahi, también como hay hijos de ex policías, hijos de ex paramilitares, hijos de exguerrilleros, también hay otros puntos de conflicto, en los indígenas, los Emberá Katio y los Emberá Chami tienen una cuestión ancestral de divergencia. (...) Entonces yo no puedo ponerme una venda y decir que todo está bien, que todo es normal, cuando yo veo que no. (Docente de artes, comunicación personal, 19 de octubre de 2018)

Así, se estableció una lista de temáticas que desde ambos colegios fueron señaladas como contenidos centrales tratados con relación a la implementación de la Cátedra de Paz, o de los espacios donde se articula la discusión sobre la construcción de paz, tal como se evidencia en el Cuadro 1. De acuerdo con los estudiantes, en el caso del IED-AJU, los temas que más se abordan con relación a la paz son: memoria histórica, prevención del acoso escolar y proyecto de vida y prevención de riesgos. En el caso de las estudiantes del colegio Santa Francisca, los temas más abordados fueron: uso sostenible de los recursos naturales, resolución pacífica de conflictos, proyectos de impacto social y justicia y Derechos Humanos. Aunque en ambos casos se reconoce que todos estos temas han sido abordados, se evidencia que, en el primer caso, dadas las condiciones del contexto en el que se inserta el colegio, la paz está más vinculada para los estudiantes con situaciones concretas que afectan su vida cotidiana; mientras que, en el segundo caso, también se da importancia a temas que no afectan directamente su cotidianidad, Y que obedecen a las temáticas de una discusión global.

Cuadro 1

Temáticas más abordadas en la Cátedra de paz

\begin{tabular}{llc}
\hline \multicolumn{1}{c}{ Temática } & IED-AJU & $\begin{array}{c}\text { Santa Francisca Ro- } \\
\text { mana }\end{array}$ \\
\hline Memoria histórica & $44 \%$ & $62 \%$ \\
Resolución pacífica de conflictos & $31 \%$ & $86 \%$ \\
Uso sostenible de los recursos naturales & $34 \%$ & $88 \%$ \\
Proyecto de vida y prevención de riesgos & $37 \%$ & $48 \%$ \\
Historia de los acuerdos de paz & $33 \%$ & $46 \%$ \\
Proyectos de impacto social & $34 \%$ & $72 \%$ \\
Prevención del acoso escolar & $37 \%$ & $67 \%$ \\
Justicia y derechos humanos & $30 \%$ & $72 \%$ \\
Participación política & $20 \%$ & $65 \%$ \\
\hline
\end{tabular}


De manera especial, en el IED-AJU tanto docentes como estudiantes inmersos en el contexto escolar, refieren problemáticas que se mueven desde lo cotidiano hasta las dinámicas más estructurales. De acuerdo con Galtung (2016), se trata de la presencia de la violencia en sus tres formas: Directa, estructural y cultural. La primera se presenta de forma visible a través de los conflictos mencionados por los entrevistados, tanto al interior del colegio como por fuera de este en sus contextos familiares y barriales. La violencia estructural se evidencia en las condiciones de pobreza y marginación, expresada a través del establecimiento en sectores de los barrios San Bernardo y Las Cruces, que han terminado funcionando como receptores de las poblaciones vulnerables que no tienen posibilidades de ubicarse en otras zonas de la ciudad. Por otro lado, se encuentra la violencia cultural, evidente en la identificación de un enemigo interno como las guerrillas, que ha marcado la planeación de las agendas gubernamentales, y genera un ambiente de polarización, pero de forma más dramática, implica la presencia constante de la violencia y discursos políticos que legitiman el exterminio de quiénes son considerados como parte del enemigo; en consecuencia, estos proyectos de nación se asientan en las representaciones de la sociedad sobre la otredad, lo diferente.

Adicionalmente, atendiendo a lo observado en el IED-AJU, dado que se trata de población "ubicada en los márgenes", se evidencia el reconocimiento de una situación de desigualdad que presenta la violencia estructural como un destino inevitable y la violencia cultural como profundamente anclada:

(...) Pero profe, yo qué hago si acá no hay opciones de trabajo, no hay nada, pues yo me voy a trabar donde el jíbaro, o yo me voy a la policía a dar plomo y después me vuelvo los de limpieza social." Otros dicen "ah no, los paracos bien porque matan a la guerrilla y a los drogadictos. O sea, es el constructo cultural el que toca cambiar, y asísuene feo, de nada va a servir la Cátedra de Paz si nuestros dirigentes y los que están arriba son absolutamente contrarios a la Cátedra de Paz, porque es que dejémonos de vainas, acá los que nos están dirigiendo para nada les interesa la paz, ni la cultura de paz. Entonces se volvió otra asignatura de relleno. (Docente de artes, comunicación personal, 19 de octubre de 2018)

Por estas razones, la Cátedra es vista como una vía para comenzar a transformar la cultura a través de combatir la presencia constante de la violencia directa, y no sólo como una herramienta para reconstruir la memoria histórica del conflicto político armado.

Trabajar para que se acaben la peleas y las riñas, los robos, las agresiones verbales, el desperdicio de la comida, hay muchas vulgaridades. (Estudiante 2, grado octavo-colegio AJU, comunicación personal, 25 de noviembre de 2018)

\subsection{Espacios alternativos de aprendizaje participativo}

Aún con esto, el tratamiento de la paz como tema central para la transformación de la realidad colombiana desde la escuela, no se ha restringido a la Cátedra de Paz. En el IED-AJU se han realizado actividades alternas como talleres, actividades culturales y conversatorios. Estas actividades han estado lideradas por las universidades, por los docentes de humanidades y ciencias sociales, los docentes que imparten la Cátedra y en menor medida por los directivos. En el caso del colegio Santa Francisca Romana, las actividades alternas fueron principalmente foros, talleres y actividades culturales, que estuvieron a cargo de los profesores de humanidades y ciencias sociales, los profesores que imparten la Cátedra y los directivos. Dichas actividades fortalecen las capacidades de los estudiantes para la comprensión de problemáticas académicas, personales y del contexto del país.

Estos espacios, aún con poca visibilización en el currículo vigente, se resaltaron en el trabajo de campo, lo que incentivó el desarrollo de talleres participativos que permitieron entender la posición de los estudiantes ante situaciones de conflicto. La estructura de los talleres siguió la metodología del "juego de roles” y se basó principalmente en las necesidades reconocidas por los estudiantes en la encuesta elaborada con antelación. La metodología del "juego de roles" permite tipificar una situación real desde la experiencia de un grupo de participantes; en esta actividad las ideas sobre los conflictos se vuelven reflexivas mediante la acción, y así permite comprender la importancia del trabajo cooperativo a nivel microsocial (salón de clase) y visualizar el territorio, el sistema educativo y el sistema familiar como elementos macrosociales.

De este modo, las actividades fueron herramientas para la identificación de estructuras simbólicas alrededor de la situación socio-política de Colombia, principalmente sobre el conflicto armado; se trabajó el acercamiento a las realidades sociales de las víctimas del conflicto y el reconocimiento de un sistema de 
actores presentes allí. Igualmente, se evidenció la manera en que afecta un contexto global a la vida cotidiana de los jóvenes y de sus familias.

Los talleres, a los que se denominaron "Piedritas de Paz" y “En sus Zapatos”, fueron espacios alternativos a las clases que permitieron abordar varias reflexiones importantes en torno a las situaciones de conflicto.

En el primer taller, desde el rol de cada uno de los participantes las reflexiones se asentaron alrededor del reconocimiento colectivo, es decir, sobre la importancia de las acciones y habilidades individuales para la consecución de un fin colectivo determinado en común acuerdo. En este tipo de experiencias se decodifica la interpretación que los participantes hacen de la realidad social a la que se enfrentan en el “juego". El espacio tuvo como resultado, la identificación del rol que cada uno tiene en la sociedad y en la construcción de paz.

En el segundo taller, que tuvo el objetivo de profundizar en los conocimientos sobre el Acuerdo de Paz y los contenidos de la Cátedra de paz, cada participante debía asumir un rol diferente al que ocupa en su entorno, lo que reflejó la capacidad de comprender las posiciones de otros actores que conviven en la sociedad con ellos. Así, de manera empática relacionaron sus roles en el marco del Acuerdo y la Cátedra, reconociendo la importancia de involucrar a todos los grupos sociales en la construcción de paz. Los estudiantes se preocuparon por entender las realidades de cada grupo (jóvenes, infancia/niñez, afrocolombianos, indígenas, población LGBTI, mujeres, campesinos) y por reconocer la diversidad y la necesidad de respeto por los derechos de todos.

Estos espacios lograron propiciar momentos de reflexión colectiva y autorreflexión por parte de los estudiantes, en la medida en que comprendieron la importancia de sus acciones desde su entorno para la construcción de una cultura de paz. Así, la capacidad de empatía con otros actores que convergen en la sociedad colombiana, reconocer sus realidades y pensar las formas de involucrar la diversidad en la búsqueda de la paz, fueron unos de los grandes resultados de los talleres.

A este respecto, es pertinente retomar el concepto de paz imperfecta, desarrollado por Francisco Muñoz (2001), desde el que es posible agrupar las "experiencias y estancias en la que los conflictos se han regulado pacíficamente, es decir en las que los individuos y/o grupos humanos han optado por facilitar la satisfacción de las necesidades de los otros" (p. 14). De este modo, se puede hallar una conexión entre "paces" de distinta índole, en el caso colombiano referidas ya sea al conflicto armado y la estructura social como a las relaciones cotidianas, se supera así la versión de la paz como algo lejano o incluso inalcanzable.

\section{Conclusiones}

La investigación realizada muestra cómo estudiantes con condiciones socioeconómicas marcadamente distintas, conciben la cultura de paz de manera diferente. Mientras quienes asisten a un colegio privado, femenino, religioso y de estratos altos, relacionan la paz con la situación sociopolítica del país, cruzada por un conflicto político armado, es decir, temas amplios que no se encuentran directamente relacionados con contextos personales; quienes asisten al colegio oficial ubicado en barrios de estratos bajos relacionan la paz con situaciones concretas de su vida cotidiana en su contexto inmediato: colegio, familia, barrio. Así, se evidencia que la preparación para la paz en el primer caso se orienta a la respuesta a una violencia considerada como estructural, que se expande a temas que no se encuentran directamente vinculados con el conflicto político armado; mientras en el segundo caso se aborda la paz como respuesta a una violencia directa, estructural y cultural, relacionada directamente con las consecuencias del conflicto armado.

En correspondencia, parece necesario que la Cátedra de Paz se oriente a los contextos específicos en los que debe implementarse, respondiendo a las demandas de la población estudiantil a la cual va dirigida. Respecto a sus contenidos se encontró precisamente que, aunque se trata de un mandato gubernamental, no existe claridad sobre los contenidos y la forma de implementación y, sin embargo, se evalúa su ejercicio. Por esta razón, los directivos y docentes de las instituciones se ven obligados a improvisar dicha implementación, acogiéndose como un tema vinculado a alguna asignatura obligatoria.

Esta situación se presenta de manera más notable en el IED-AJU, donde se resalta que la Cátedra de Paz no se presenta como un tema transversal al currículo escolar, ni tampoco como una asignatura exclusiva. 
En el colegio Santa Francisca Romana, por el contrario, se insiste en su transversalidad, pero la falta de claridad sobre su implementación lleva a que se incluyan y enfaticen problemáticas distantes del conflicto político armado.

A su vez, los docentes motivados por las necesidades de la población estudiantil optan por realizar actividades en el marco de las asignaturas que les corresponden, con el propósito de incidir en la transformación de las relaciones conflictivas y violentas entre los estudiantes o como forma de incentivar las competencias de sus estudiantes en materia de conocimiento y concientización sobre la situación sociopolítica del país. En conexión con el diagnóstico que hace Sánchez (2015), es importante reflexionar respecto a la noción de paz imperfecta (ya sea formulada con tal nombre o a través de otras palabras) cuando afirma que: "en los espacios educativos en Colombia donde se ha planteado este concepto, se ha observado que ha ayudado a un grupo de maestros y maestras a que no pierdan el ánimo de desarrollar sus metodologías de paz, aun cuando los resultados en las aulas sean parciales” (p. 71)

Finalmente, se reconoce que es necesario seguir trabajando en el desarrollo de mecanismos y estrategias educativas más allá de los espacios institucionalizados, por ejemplo, los talleres realizados; a partir de estos, se logra generar autorreflexión y reconocimiento de la importancia y del respeto hacia el otro como primer paso para transformar contextos violentos en contextos pacificados, donde la presencia constante de conflictos no implicará la presencia de relaciones violentas.

\section{Referencias}

Álvarez, J. (2016). La transformación del barrio Las Cruces y su consolidación como borde urbano durante el siglo XX [Tesis de Maestría de la Universidad Nacional de Colombia]. Archivo de la Universidad Nacional de Colombia. http://bdigital.unal.edu.co/55496/7/1032439504.2016.pdf

Redacción de El Tiempo. (2016, 28 de mayo). El operativo sin precedentes que se tomó la olla del ‘Bronx’. El Tiempo. https:/www.eltiempo.com/archivo/documento/CMS-16606031

Boqué, M. C, Pañellas V. M., Alguacil de Nicolás, M., García, L. (2014). La cultura de paz en la educación para la ciudadanía y los derechos humanos en los libros de texto de educación primaria. Perfiles Educativos, 36(146), 80-97.

Bourdieu, P. y Passeron, J. C. (1979). La reproducción. Elementos para una teoría del sistema de enseñanza. Laia.

Carvalho, M. E., Morais, G., Carvalho, B. K. (2019). Dos castigos escolares à construção de sujeitos de direito: Contribuições de políticas de direitos humanos para uma cultura da paz nas instituições educativas. Políticas Públicas Educativas, 27(102), 24-46. https://doi.org/10.1590/s0104-40362018002601366

Castellano, M. C., Virviescas, J., Castro, E., Alvarino, C., Pinzón F. y Gutiérrez, R. (2017). Resolución de conflictos para el fomento de la cultura de paz: Importancia de los medios de comunicación alternos en Colombia. Revista Lasallista de investigación, 14(1), 56-65.

Cepeda, I. (2016). La cultura de paz como un proceso que se teje entre el pensar y el actuar. Andamios, 13(32), 411-414.

Colombia 2020. (2018, junio 23). La reducción de muertes en el conflicto después del acuerdo con las Farc. El Tiempo. https://colombia2020.elespectador.com/pais/la-reduccion-de-muertes-en-el-conflicto-despues-del-acuerdo-con-las-farc

Galtung, J. (2016). La violencia cultural, estructural y directa. Cuadernos de Estrategia, 183, 147-168.

García, E. (2014). La capacitación mediática e informativa como contenido de la educación para la paz. Historia y Comunicación Social, 19, 547-558. https://doi.org/10.5209/rev_HICS.2014.v19.45048

Giddens, A. (2004). Sociología. Alianza.

Martínez, M. (2017). Potenciando el enfoque de la educación para la paz en la enseñanza universitaria española: Una propuesta de actividades en asignaturas de lengua inglesa, Íkala, Revista de Lenguaje y Cultura, 22(1), 87-100. https://doi.org/10.17533/udea.ikala.v22n01a06

Moreira, G., Córdova, J., Lalangui, D. (2016). La cultura de paz y el papel de los comunicadores en la resolución de conflictos. Acuerdo de paz entre Ecuador y Perú. Revista Espacios, 37, 1-8. 
Moreira, L. y Branco, A. (2016). Proceso de socialización y promoción de la cultura de paz en la perspectiva de policías militares. Estudios de Psicología, Campinas, 33(3), 553-563. https://doi.org/10.1590/1982-02752016000300018

Muñoz, F. A. (Ed.). (2001). La paz imperfecta, Universidad de Granada.

Navia, J. (1998, 6 de marzo). San Bernardo: Un barrio atrapado y sin salida. El Tiempo. https://www.eltiempo.com/archivo/documento/MAM-750538

Ramírez, H. C. (2017). Aportaciones de la educación musical a la educación para la paz. Buscando la transverdad en la era de la posverdad. Revista Electrónica Complutense de Investigación en Educación Musical, 14,129-151. https://doi.org/10.5209/RECIEM.54777

Redacción de El Tiempo (1992, 25 de enero). Cedritos, un conjunto abierto de historias. El Tiempo. https://www.eltiempo.com/archivo/documento/MAM-20777

Redacción Portafolio. (2010, 29 de octubre). Cedritos: Integración de vivienda y comercio. Portafolio. https://www.portafolio.co/economia/finanzas/cedritos-integracion-vivienda-comercio-255304

Salazar, L. y Boschi da Silva, H. (2014). Gênese discursiva da fórmula 'cultura de paz'. Acta Scientiarum. Language and Culture, 36(2), 131-137. https://doi.org/10.4025/actascilangcult.v36i2.22567

Sánchez, S. y Vargas, M. (2017). La cultura de paz en educación secundaria obligatoria. Estudio comparado de libros de texto de educación para la ciudadanía. Bordón. Revista de Pedagogía. 69(2), 115-130.

https://doi.org/10.13042/Bordon.2016.49768

Santos, C. J. (2016). Cultura de paz, educomunicación y TIC en Colombia. Opción, 32(12), 609-637.

Sánchez C. M. (2015). Educación para la cultura de la paz. Una aproximación psicopedagógica. Ediciones USTA.

Serrano, G. A. (2015, 12 de noviembre). Indigencia y drogas deterioran al tradicional barrio San Bernardo. El Tiempo. https://www.eltiempo.com/archivo/documento/CMS-16429413

Vargas, A. D. (2016). Espacio de investigación para la educación de la paz y la convivencia apoyado por las TIC. Opción, 32(8), 920-935.

\section{Breve CV de las autoras}

\section{Catalina Acosta Oidor}

Socióloga de la Universidad del Valle (2009) y Magíster en Sociología de la Facultad Latinoamericana de Ciencias Sociales, Flacso (2015). Actualmente, docente de la Facultad de Sociología de la Universidad Santo Tomás, Bogotá. Líder del grupo de investigación Estudios Interdisciplinarios de la Sociedad y la Cultura. Publicación más reciente: Acosta, C. (2019). La reinvención de la violencia en Colombia como desafío para la pacificación: el caso de las bacrim, en Sánchez y Acosta (Eds.) (2019). Las Huellas del Desarrollo. Intersecciones entre conflicto, reconfiguración social y pacificación en Colombia. Bogotá: Ediciones USTA. 323 -354. Email: catalinaacosta@usantotomas.edu.co

ORCID ID: https://orcid.org/0000-0003-0823-7021

\section{Luz Ángela Tabares Rojas}

Socióloga de la Universidad de Toulouse II (2004), Magíster en Economía solidaria e innovación (2006) y Sociología aplicada a la gestión local (2007). Experiencia en la gerencia de programas socio-económicos y en la aplicación responsable de metodologías y herramientas a proyectos de intervención y de generación de ingresos. Integrante del grupo de investigación Estudios Interdisciplinarios de la Sociedad y la Cultura. Con interés en temáticas de economía popular, social y solidaria, socio economía y desarrollo local. Actualmente, fundadora del proyecto Aglaïa Coaching Creativo, que busca impulsar el emprendimiento con impacto en el entorno. Email: luzangela.tabares@gmail.com

ORCID ID: https://orcid.org/0000-0002-5930-7096 


\section{Paula Natalia Castillo Acosta}

Socióloga, de la Universidad Santo Tomás, integrante del semillero Educación y Política y del grupo de investigación Estudios Interdisciplinarios de la Sociedad y la Cultura. Con interés y experiencia investigativa en juventud, representaciones sociales, educación y educación para la paz. Con experiencia e interés académico en temas como la sociología de la educación, educación para la paz, jóvenes y partidos políticos. Publicación más reciente: Castillo, P. (2017) Juventudes partidarias: aproximación a los factores y/o motivaciones que intervienen en su consolidación. En Libro de resúmenes, 670, Asociación Latinoamericana de Sociología (ALAS). Email: paulacastilloa@usantotomas.edu.co

ORCID ID: https://orcid.org/0000-0003-2789-6495

\section{María Catalina López Andrade}

Socióloga de la Universidad Santo Tomás, integrante del semillero Educación y Política y del grupo de investigación Estudios Interdisciplinarios de la Sociedad y la Cultura. Con experiencia e interés académico en la sociología de la educación, estudios de género, cultura de paz, educación para la paz, víctimas de conflicto y de violencia por condición de género, juventud y pobreza. Publicaciones recientes: Capitulo de libro: Ortiz, A. M., y otros (2018). Deconstruyendo la educación para la paz a partir de la experiencia I.E.D Antonio José Uribe. En USTA. (2018). Simposio de investigación USTAMED, Universidad Santo Tomás, Medellín. Email: marialopeza@usantotomas.edu.co

ORCID ID: https://orcid.org/0000-0002-9840-1114

\section{Luisa Fernanda Luque Ramírez}

Socióloga de la Universidad Santo Tomás, Bogotá, integrante del semillero Educación y política, del semillero Degenerando los géneros y del grupo de investigación Estudios interdisciplinarios de la sociedad y la cultura de la misma universidad. Con interés y experiencia investigativa en educación y medio ambiente; educación y paz; pedagogías alternativas, nuevas metodologías educativas; género y masculinidades; teoría del género; género y cultura; género y estética y teorías feministas. Última publicación: ¿Cómo están viviendo los colombianos el COVID-19? (2020) Revista Las dos orillas. Email: luisa.luque@ usantotomas.edu.co

ORCID ID: https://orcid.org/0000-0002-7077-2263

\section{Ana María Ortiz Arévalo}

Socióloga de la Universidad Santo Tomás, Bogotá, integrante del semillero Educación y política, del semillero Degenerando los géneros y del grupo de investigación Estudios interdisciplinarios de la sociedad y la cultura de la misma universidad. Publicaciones recientes: Capitulo de libro: Ortiz, A. M., y otros (2018). Deconstruyendo la educación para la paz a partir de la experiencia I.E.D Antonio José Uribe. En USTA. (2018). Simposio de investigación USTAMED, Universidad Santo Tomás, Medellín. Líneas de interés investigativo: Educación y género, masculinidades, educación experiencial, diversidades sexuales y educación para la paz. Email: anaortiza@usantotomas.edu.co

ORCID ID: https://orcid.org/0000-0003-3432-8752

\section{Nataly Vargas Rodríguez}

Socióloga de la Universidad Santo Tomás y miembro activa en el Semillero de Educación y Política. Acompañamiento en las estrategias pedagógicas Educación para la generación de una cultura de paz (2018), La educación popular como alternativa para la comprensión de la realidad en Colombia (2018), y en los espacios de participación ciudadana Las artes escénicas como estrategia para la generación de una cultura de paz (2018). Últimas publicaciones: ¿Cómo están viviendo los colombianos el COVID-19? (2020) Revista Las dos orillas y El feminismo entendido desde los hombres (2020) en Congreso Internacional de Jóvenes Investigadores ScienceTubers. Email: nataly.vargas@usantotomas.edu.co

ORCID ID: https://orcid.org/0000-0001-5543-4576 